\title{
GADD45B mediates podocyte injury in zebrafish by activating the ROS-GADD45B-p38 pathway
}

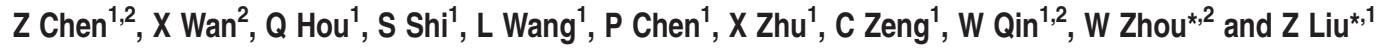

GADD45 gene has been implicated in cell cycle arrest, cell survival or apoptosis in a cell type specific and context-dependent manner. Members of GADD45 gene family have been found differentially expressed in several podocyte injury models, but their roles in podocytes are unclear. Using an in vivo zebrafish model of inducible podocyte injury that we have previously established, we found that zebrafish orthologs of gadd45b were induced upon the induction of podocyte injury. Podocyte-specific overexpression of zebrafish gadd45b exacerbated edema, proteinuria and foot-process effacement, whereas knockdown of gadd $45 \mathrm{~b}$ by morpholino-oligos in zebrafish larvae ameliorated podocyte injury. We then explored the role of GADD45B induction in podocyte injury using in vitro podocyte culture. We confirmed that GADD45B was significantly upregulated during the early phase of podocyte injury in cultured human podocytes and that podocyte apoptosis induced by TGF- $\beta$ and puromycin aminonucleoside (PAN) was aggravated by GADD45B overexpression but ameliorated by shRNA-mediated GADD45B knockdown. We also showed that ROS inhibitor NAC suppressed PAN-induced GADD45B expression and subsequent activation of p38 MAPK pathway in podocytes and that inhibition of GADD45B diminished PAN-induced p38 MAPK activation. Taken together, our findings demonstrated that GADD45B has an important role in podocyte injury and may be a therapeutic target for the management of podocyte injury in glomerular diseases.

Cell Death and Disease (2016) 7, e2068; doi:10.1038/cddis.2015.300; published online 21 January 2016

Podocyte dysfunction, injury or loss is a common and decisive cause of various glomerular diseases and understanding the molecular mechanism underlying podocyte response to stress will be very helpful to undermine the pathogenesis of podocyte injury and the targeted therapy for glomerular diseases.

The members of Gadd45 gene family, Gadd45a, Gadd45b and Gadd45r have been commonly implicated in stress signaling in response to physiological or environmental stressors, resulting in cell cycle arrest, DNA damage repair, cell survival, senescence and apoptosis. ${ }^{1}$ Recently, this gene family has been found differentially expressed in several podocyte injury models. Zhang et al. ${ }^{2}$ observed an induction of $G A D D 45 \beta$ mRNA expression by lipopolysaccharide in the lung, kidney and spleen, which had the highest GADD45 $\beta$ mRNA expression among all of the tissues examined. Jeffrey $W$ Pippin reported that protein expression of GADD45 was increased in glomeruli from passive Heymann nephritis rats and cultured podocytes exposed in vitro to C5b-9. ${ }^{3}$ More recently, Shi et al. ${ }^{4}$ reported that Gadd45b was upregulated in glomeruli of mice with podocyte-specific deletion of Dicer, suggesting the involvement of Gadd45b in podocyte injury. However, no functional characterization of Gadd45 genes in podocytes has been conducted to date and the role of GADD45B in the context of podocyte injury remains unclear.

Zebrafish has emerged as a new vertebrate model system for renal glomerular research. The podocytes and renal glomeruli in zebrafish kidney are structurally, molecularly and functionally conserved, rendering zebrafish a valuable and relevant model for podocyte studies. To characterize the role of GADD45b in podocyte injury, we therefore employed zebrafish as an in vivo model system and human podocytes as an in vitro model. We observed the upregulation of GADD45B on podocyte injury in zebrafish renal glomeruli as well as in cultured human podocytes treated with TGF- $\beta$ and PAN. We further showed that podocyte-specific overexpression of zebrafish orthologs of gadd45b predisposed podocytes to injury, whereas inhibition of gadd45b expression in zebrafish larvae ameliorated podocyte injury and reduced proteinuria. Furthermore, we found that the ROS-GADD45B-p38 pathway was involved in the regulation of GADD45B expression and deleterious role in podocyte injury. Collectively, we have identified GADD45B as an important player in podocyte injury.

\footnotetext{
${ }^{1}$ National Clinical Research Center of Kidney Diseases, Jinling Hospital, Nanjing University School of Medicine, Nanjing, China and ${ }^{2}$ Department of Pediatrics and Communicable Diseases, University of Michigan Medical School, Ann Arbor, MI, USA

${ }^{*}$ Corresponding author: W Zhou, Department of Pediatrics and Communicable Diseases, University of Michigan Medical School, 8220E MSRB III, 1150 West Medical Center Drive, Ann Arbor, Ml 48109-5646, USA. Tel: +1 734647 9429; Fax: +1 734615 1386; E-mail: weibinz@med.umich.edu

or Z-H Liu, National Clinical Research Center of Kidney Diseases, Jinling Hospital, Nanjing, University School of Medicine, 305 East Zhongshan Road, Nanjing 210002, China. Tel: +86 25 80860218; Fax: +86 25 84801992; E-mail: liuzhihong@nju.edu.cn

Abbreviations: DMSO, dimethyl sulfoxide; ERK, extracellular signal-regulated kinase; GADD45, growth arrest and DNA damage gene 45; Gal4, galactose-responsive transcription factor GAL4; JNK, c-Jun N-terminal kinase, JNK; Ifabp, liver fatty-acid binding protein; LPS, lipopolysaccharide; MAPK:, mitogen-activated protein kinase; MO, morpholino; MTZ, metronidazole; NTR, nitroreductase; PAN, puromycin aminonucleoside; ROS, reactive oxygen species; TGF- $\beta$, transforming growth factor- $\beta$; UAS, upstream active sequence; VDBP, vitamin D-binding protein

Received 28.5.15; revised 25.8.15; accepted 10.9.15; Edited by T Kaufmann
} 


\section{Results}

gadd $45 \mathrm{ba} / \mathrm{b}$ expression is upregulated in zebrafish with podocyte injury. We isolated podocytes from kidney of $T g$ (pod:Gal4;UAS:NTR-mcherry) zebrafish and confirmed the isolated podocytes had enriched podocin expression (Figure 1a). gadd45ba/bb mRNA expression were detected on the isolated podocytes (Figure 1b). We observed GADD45B expression on glomeruli of human biopsy and found that GADD45B signals are very weak in glomeruli of normal kidney tissues, whereas GADD45B expression in FSGS patients was significantly increased as compared with normal control group (Figures 1c and d).

Previously, we have established a transgenic zebrafish model of inducible podocyte injury, in which a bacterial nitroreductase (NTR) is specifically expressed under the control of zebrafish podocin promoter. ${ }^{5}$ In this transgenic zebrafish, metronidazole (MTZ) treatment can induce podocyte foot-process effacement, podocyte apoptosis, edema and proteinuria. Here, we refined this model of podocyte injury using a double transgenic zebrafish Tg(pod:Gal4;UAS:NTR$m$ Cherry), in which transcription factor Gal4 was specifically expressed in podocytes, and NTR-mCherry was under the control of UAS promoter. In this model, renal glomeruli could be conveniently isolated based on the mCherry fluorescence and podocyte injury induced by MTZ treatment (Figure 2a). We treated the double transgenic zebrafish with MTZ for 12 and $24 \mathrm{~h}$ followed by the isolation of zebrafish glomeruli under fluorescence microscopy. qRT-PCR results showed that the mRNA expression of both zebrafish orthologs of GADD45B (gadd45ba and gadd45bb) were significantly upregulated at as early as $12 \mathrm{~h}$ post treatment, when nephrin expression was barely decreased (Figures $2 \mathrm{~b}-\mathrm{d}$ ). After $24 \mathrm{~h}$ of MTZ treatment, nephrin expression was significantly decreased, whereas the elevated mRNA expression of both gadd $45 \mathrm{ba}$ and gadd $45 \mathrm{bb}$ persisted. Thus, gadd $45 \mathrm{~b}$ was induced at the early phase of podocyte injury prior to the downregulation of nephrin, suggesting it is an early mediator of podocyte injury. In addition, we found that gadd45ba expression was more abundant than gadd $45 b b$ in normal zebrafish glomeruli and the induction of gadd45ba by podocyte injury was more prominent than that of gadd45bb (Figures $2 \mathrm{c}$ and d), suggesting gadd45ba may have a major role in zebrafish podocytes compared with gadd $45 \mathrm{bb}$.

gadd45ba/b overexpression aggravates MTZ-induced podocyte injury and proteinuria. Given that gadd $45 \mathrm{ba} / \mathrm{b}$ expression was induced in zebrafish podocyte injury, we sought to functionally characterize gadd $45 \mathrm{ba} / \mathrm{b}$ in podocyte injury. First, we generated transgenic zebrafish lines stably expressing gadd45ba or gadd $45 \mathrm{bb}$ in podocytes using the binary Gal4/UAS system (Figure 3a). GFP expression in cardiomyocytes allowed for easy identification of the $T g$ (UAS:gadd45ba/b,cm/c2:GFP) transgenic fish. When $T g$ (UAS:gadd45ba/b,cm/c2:GFP) was mated with $T g($ pod: Gal4;UAS:NTR-mCherry), podocin-promoter-driven Gal4 expression could activate both NTR-mCherry and gadd $45 \mathrm{ba} / \mathrm{b}$ expression exclusively in podocytes, which was confirmed by whole-mount in situ hybridization (Figure 3a).

The transgenic fish Tg(pod:Gal4;UAS:NTR-mCherry;UAS: gadd45ba/b,cm/c2:GFP) appeared normal and fertile, without any edema. To determine the role of gadd $45 \mathrm{ba} / \mathrm{b}$ in podocyte

b
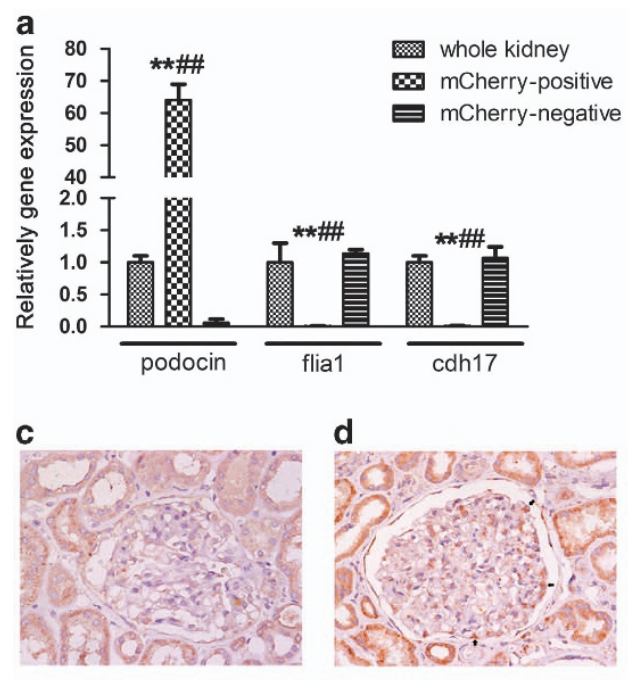
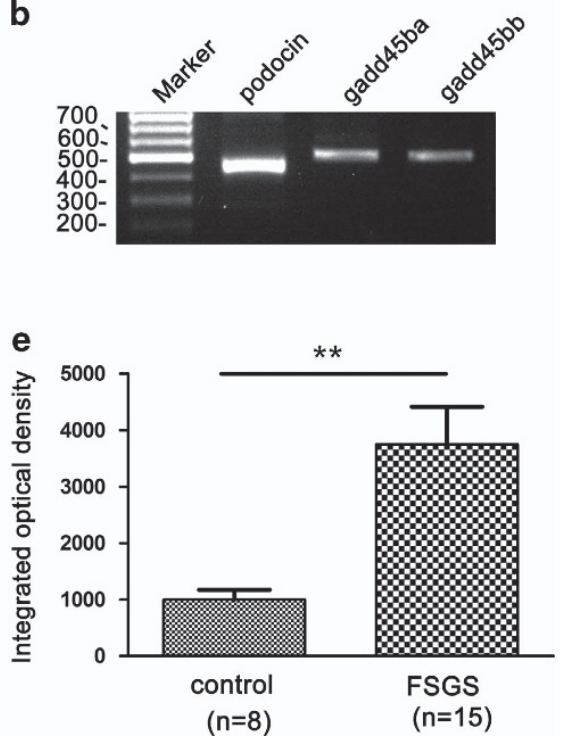

Figure 1 Validation of gadd45ba/bb expression on podocyte. (a) qRT-PCR validation of isolated podocytes. qRT-PCR analysis confirmed that the expression of podocyte marker podocin in isolated podocytes (mCherry positive) was highly enriched as compared with those from whole kidney and mcherry-negative cells. In contrast, expression of endothelial marker flia1 and tubular marker cdh17 were significantly lower in isolated podocytes as compared with those in whole kidney and mCherry-negative cells. Results are represented as mean \pm S.D. $(n=3),{ }^{\star \star} P<0.01$ compared with whole kidney, ${ }^{\#} P<0.01$ compared with mcherry-negative cells. (b) RT-PCR analysis of gadd45ba/bb mRNA expression in isolated podocytes. gadd45ba/bb expressed in isolated podocytes which express podocin (c, d) GADD45B expression in biopsy of normal control (c) and FSGS patient (d) by immunohistochemical staining. GADD45B signals are very weak in glomeruli of normal kidney tissues, whereas GADD45B expression in FSGS patients was significantly increased as compared with normal control group. Arrows indicate GADD45B expression on podocytes of FSGS patient. Original magnification $\times 400$ (e) Semiquantitative analysis of immunohistochemical staining results showed GADD45B expression of glomeruli of FSGS patients were significantly higher than the control group. Results are represented as mean \pm S.D. ${ }^{* *} P<0.01$ 
a
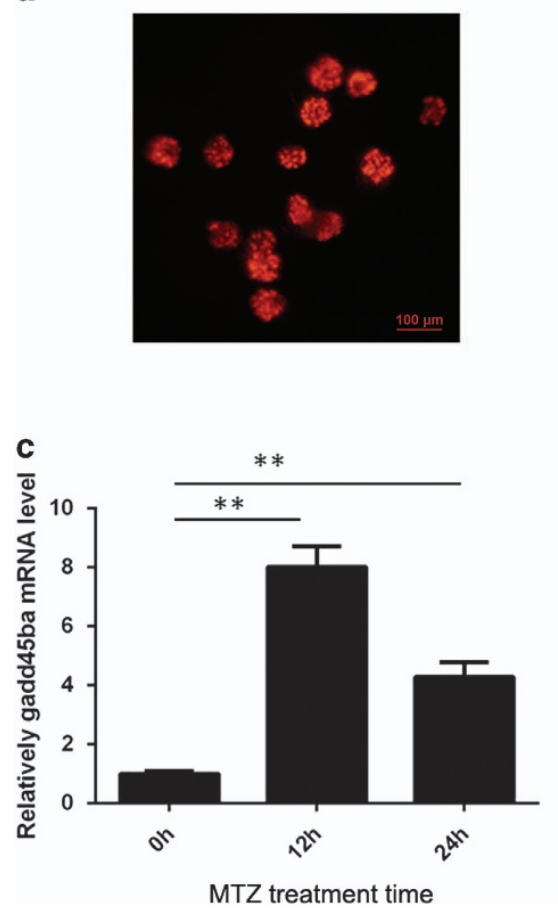

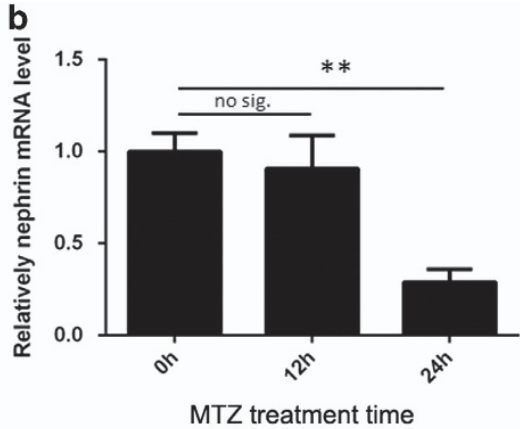

d

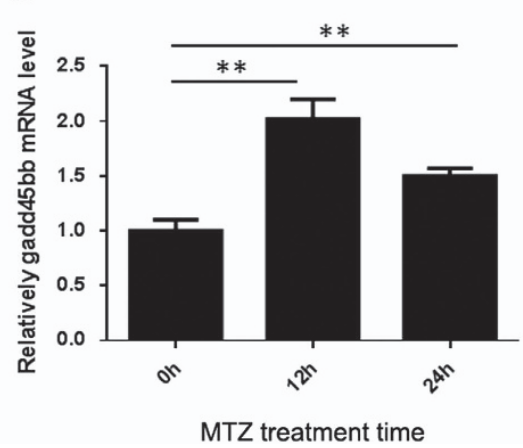

Figure 2 gadd45ba and gadd45bb are induced by podocyte Injury in zebrafish. (a) Dissected renal glomeruli from Tg(pod:gal4;UAS:NTR-mcherry), mCherry expression in podocytes allow for easy collection under fluorescence microscope. (b-d) Quantitation of nephrin (b), gadd45ba (c) and gadd45bb (d) transcripts by quantitative RT-PCR before and after MTZ $(120 \mu \mathrm{M})$ treatment. Results are represented as mean \pm S.D. $(n=3),{ }^{* *} P<0.01$

injury, we then induced podocyte injury with MTZ after sorting the transgenic embryos by the expression of fluorescent proteins. Larvae with GFP fluorescence in the heart and mCherry fluorescence in glomeruli expressed both gadd45ba/b and NTR-mCherry in podocytes, whereas larvae with mCherry fluorescence in glomeruli only expressed NTR-mCherry in podocytes and hence as controls (Figure 3b). As MTZinduced podocyte injury causes a very unique periorbital edema (POE) phenotype in zebrafish larvae, resembling nephrotic syndrome in human patients (Figure 3c, Supplementary Figure 1), we categorized the fish with POE into two subgroups according to the severity of edema. The mild group had mild POE alone with moderate foot-process effacement but the severe group displayed severe POE and whole-body edema with more extensive foot-process effacement (Figure 3c). By quantifying the percentage of zebrafish larvae with POE, we found that MTZ could induce POE in zebrafish carrying NTR-mCherry in a dosage-dependent manner. In addition, gadd45ba/b overexpression in zebrafish podocytes significantly increased the penetrance of MTZ-induced POE by $\sim 20 \%$ (Figure $3 d$, Supplementary Table 1). Indeed, gadd45ba/b overexpression in podocytes significantly aggravated the severity of edema induced by MTZ (Figure 3e). Although the percentage of mild edema group was not significantly changed, the percentage of severe edema group was drastically increased by gadd45ba/b overexpression. It is also notable that the effect of gadd45ba overexpression was more prominent than that of gadd $45 b b$ overexpression (Figures 3d and e). In conclusion, excessive gadd45ba/b in podocytes aggravated podocyte injuries.
To test whether overexpression of gadd $45 \mathrm{ba} / \mathrm{b}$ exacerbates proteinuria, we performed proteinuria assay using a previously established Tg(Ifabp:VDBP-GFP) zebrafish expressing GFP-tagged vitamin D-binding protein (VDBP). ${ }^{5}$ In this transgenic fish, VDBP-GFP, which has a molecular weight (MW) and isoelectric point (PI) (MW:79.6 kD; PI 5.67) comparable to human albumin (MW:65.5; Pl: 5.97), serves as a tracer plasma protein for both size and charge selection of glomerular filtration barrier. Disruption of the glomerular filtration barrier by MTZ-induced podocyte injury in Tg(pod:Gal4; UAS-NTRmCherry; Ifabp:VDBP-GFP) led to strong accumulation of GFP fluorescence in the proximal tubules, as VDBP-GFP is reabsorbed by tubular epithelium (Figure 4a). Meanwhile, mCherry fluorescence was present in the proximal tubules, presumably due to the tubular reabsorption of mCherry protein released from disintegrated podocytes (Figure 4a). Using ELISA against GFP to measure the excretion of VDBP-GFP into water by the transgenic larvae, we found that overexpression of gadd $45 \mathrm{ba} / \mathrm{b}$ in podocytes significantly increased proteinuria induced by $24 \mathrm{~h}$ of MTZ treatment (Figure $4 \mathrm{~b}$, Supplementary Table 2). Noticeably, without MTZ treatment, Tg(pod:Gal4;UAS:NTR-mCherry;UAS:gadd45ba/b;/fabp: VDBP-GFP) did not show any significant excretion of GFP either, indicating that gadd $45 \mathrm{ba} / \mathrm{b}$ overexpression alone was not sufficient to impair the glomerular filtration barrier. These proteinuria data were consistent with the tubular accumulation of GFP and the edema phenotype.

gadd45ba/b overexpression aggravates MTZ-induced podocyte apoptosis. To investigate the effect of gadd $45 \mathrm{ba} / \mathrm{b}$ overexpression on podocyte apoptosis, we performed 
b

a

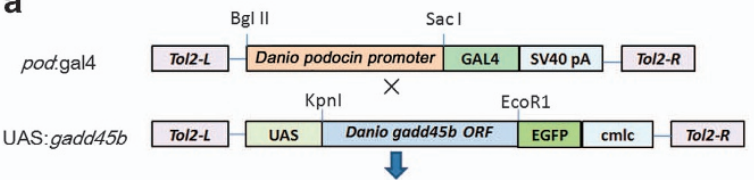

Podocyte specific expression of gadd $45 \mathrm{~b}$ transgenic fish

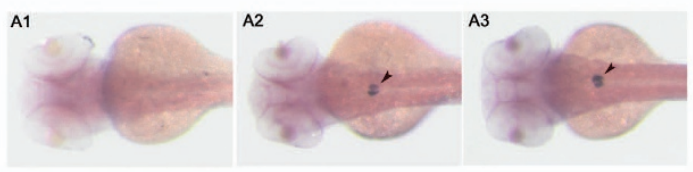

C

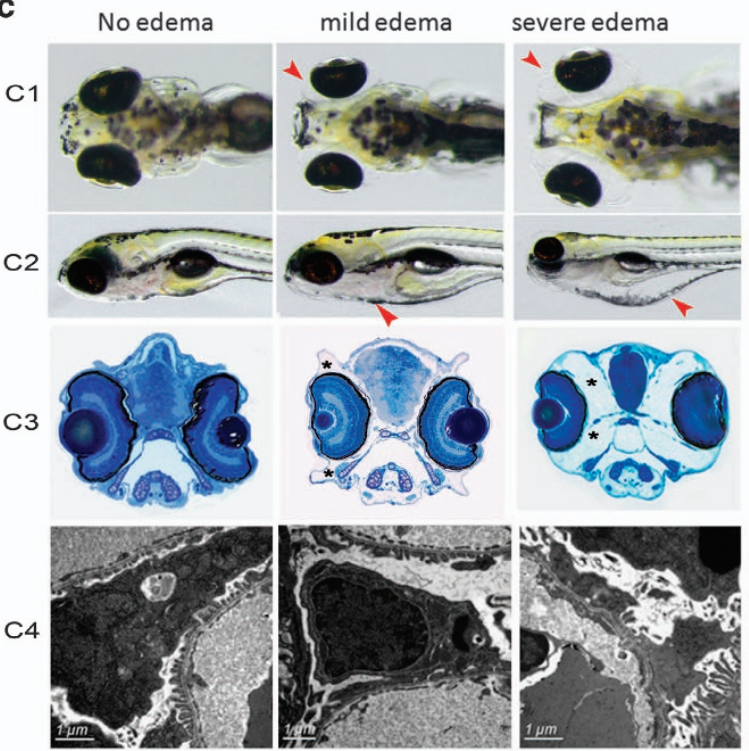

d

e

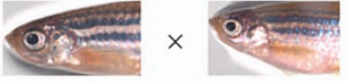

Tg(UAS:gadd45b) , Tg(Pod:gal4;UAS:NTR-mcherry)

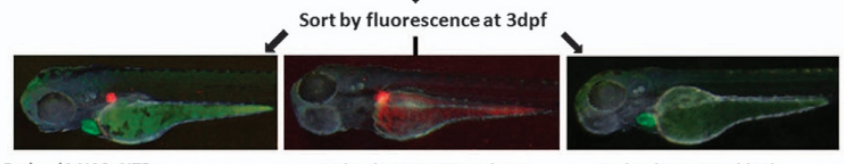

Pod:gal4;UAS: NTRmcherry;UAS:gadd45b,crp/c:gfp

Pod:gal4;UAS NTR-mcherry Pod:gal4;UAS:gadd45b
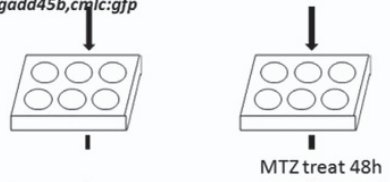

1

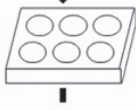

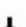

Observe Edema, Histology and Apoptosis
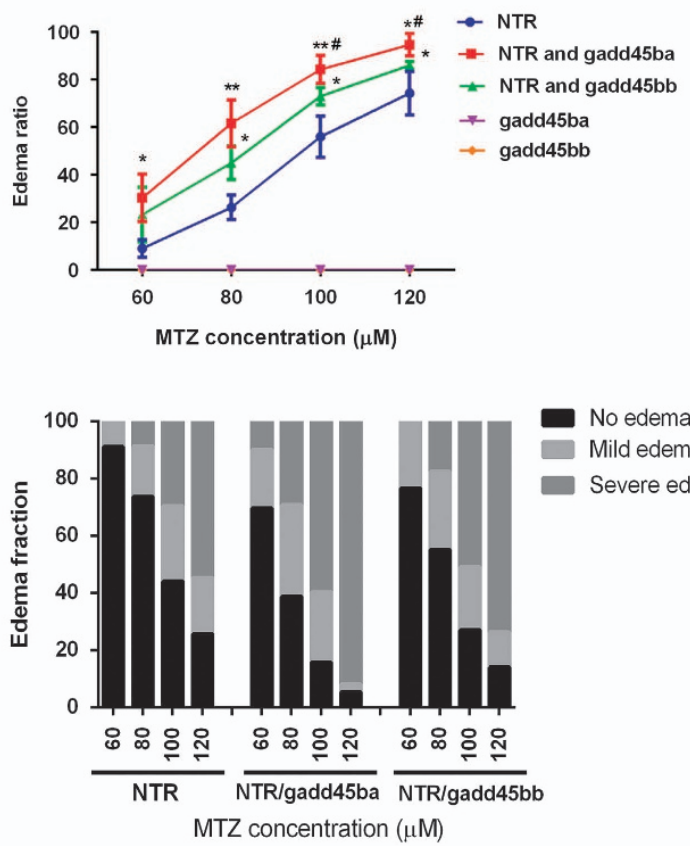

Figure 3 gadd45ba/b overexpression in podocytes aggravates MTZ-induced podocyte injury. (a) Schematic graph showing the generation of transgenic fish. Podocyte-specific expression of gadd45ba/b is confirmed by in situ hybridization in WT (A1), $T g$ (pod:Gal4,UAS:gadd45ba) (A2) and Tg (pod:Gal4,UAS:gadd45bb) (A3) at 3dpf. (b) Experimental scheme to test gadd45ba/b overexpression on MTZ-induced podocyte injury. (c) Representative figures showing the phenotypes due to MTZ-induced podocyte injury. (c1) Dorsal view of five dpf larvae showing periorbital edema (red arrowhead). (c2) side view of five dpf larvae showing whole-body edema (red arrowhead). (c3) Crosssection of eyes of five dpf larvae stained with methylene blue showing the severity of periorbital edema (asterisks). (c4) TEM showing the degree of foot-process effacement. (d) Quantitation of the percentage of larvae with periorbital edema. The nonfluorescence fish were used as negative controls and had no observable periobital edema phenotypes after being treated with MTZ. (e) Quantitation of the percentage of larvae with mild and severe edema. Results are represented as mean \pm S.D. $(n=3)$, ${ }^{*} P<0.05,{ }^{* *} P<0.01$ versus NTR group, ${ }^{\#} P<0.05$ versus NTR and gadd45bb group

immunostaining of cleaved caspase-3, an essential mediator of apoptosis, on zebrafish pronephric glomeruli. We observed that apoptosis was significantly increased following MTZ treatment in the pronephric glomeruli of gadd $45 \mathrm{ba} / \mathrm{b}$ overexpression fish than those of the control group expressing NTR-mCherry alone in podocytes (Figure 5).

Inhibition of gadd45ba/b expression alleviates MTZinduced podocyte injury and proteinuria. Based on the aforementioned results, we wondered whether inhibition of gadd $45 \mathrm{ba} / \mathrm{b}$ in zebrafish could reduce MTZ-induced podocyte injury. To answer this question, we utilized morpholino-oligos specifically blocking the mRNA splicing of either gadd45ba or gadd45bb (Figure 6a). The efficacy of these MOs was confirmed by RT-PCR and sequencing, showing that gadd $45 \mathrm{ba}-\mathrm{MO}$ and gadd $45 \mathrm{bb}-\mathrm{MO}$ could block the splicing of adjacent introns (Figure 6b). qRT-PCR results showed that gadd45ba-MO resulted in $47.1 \pm 6.8 \%$ (3dpf) and $25.3 \pm 8.5 \%$ (5dpf) reduction in gadd45ba mRNA level, whereas gadd $45 \mathrm{bb}-\mathrm{MO}$ resulted in $42.4 \pm 5.9 \%$ (3dpf) and $19.2 \pm 7.6 \%$ (5dpf) in gadd $45 b b$ mRNA level (Figures $6 \mathrm{c}$ and d). Injection of either gadd45ba-MO or gadd45bb-MO did not 

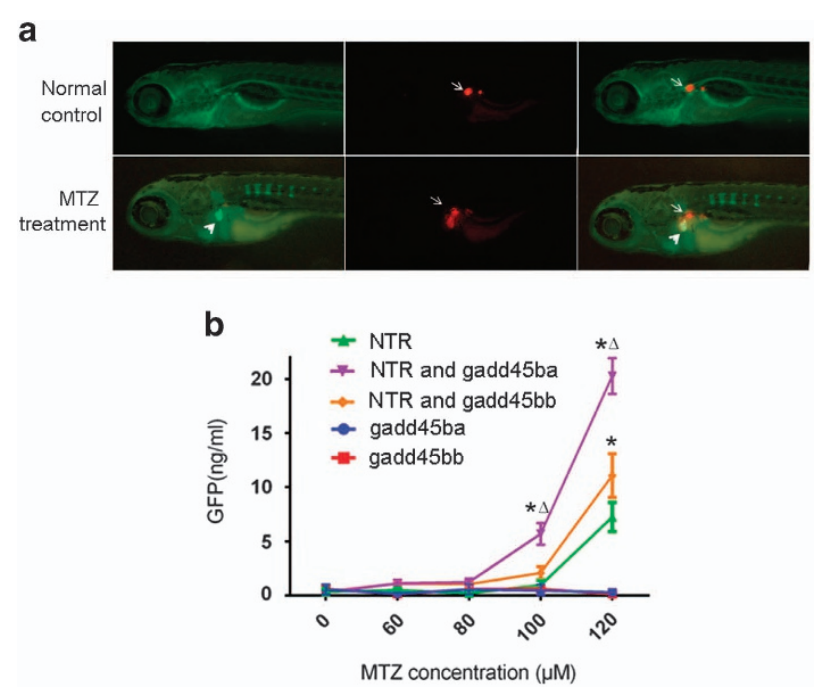

Figure 4 gadd45ba and gadd45bb overexpression in podocytes aggravates MTZ-induced proteinuria. (a) Representative fluorescence figures showing disruption of the glomerular filtration barrier by MTZ leads to accumulation of GFP fluorescence in the proximal tubules (arrowheads) in Tg(pod:Gal4;UAS-NTR-mCherry; Ifabp: VDBP-GFP). mCherry fluorescence (arrows) is reduced in glomeruli and accumulated in proximal tubules presumably due to podocytes loss. (b) Quantitation of GFP by ELISA showing zebrafish expressing gadd45ba/b in podocytes exhibit more poteinuria induced following MTZ-induced podocyte injury. Results are represented as mean \pm S.D. $(n=3),{ }^{*} P<0.01$ versus NTR group; $\Delta P<0.01$ versus NTR and gadd $45 \mathrm{bb}$ group cause any embryonic developmental defect prior to the induction of podocyte injury. gadd45ba-MO or gadd45bb-MO could, however, significantly alleviate MTZ-induced POE while control-MO did not impose such an effect (Figure 6e, Supplementary Table 3). Interestingly, the effect on podocyte injury by gadd45ba knockdown is more pronounced than gadd $45 b b$, consistent with the finding that overexpression of gadd45ba had a greater effect in aggravating podocyte injury than gadd $45 \mathrm{bb}$. Furthermore, knockdown of gadd45ba/b could also significantly lower the MTZ-induced proteinuria in our zebrafish models (Figure 6f, Supplementary Table 4).

GADD45B overexpression sensitizes podocytes to TGF- $\beta$ and PAN-induced apoptosis. Podocyte deleterious factors, such as TGF- $\beta$ and PAN can induce GADD45B expression, which preceded the decrease of NEPHRIN protein level induced by TGF- $\beta$ and PAN treatment (Figures $7 \mathrm{a}$ and $\mathrm{b}$ ). To determine the effect of GADD45B upregulation on podocyte injury, GADD45B was overexpressed in cultured human podocytes, which was confirmed by western blot (Figure 7c). Podocytes with GADD45B overexpression had a significantly higher percentage of Annexin V-positive apoptotic cells compared with the mock-transfected control cells in the absence or presence of TGF- $\beta$ or PAN, suggesting that GADD45B contributes to apoptosis in podocytes (Figures $7 \mathrm{e}$ and g). Consistently,

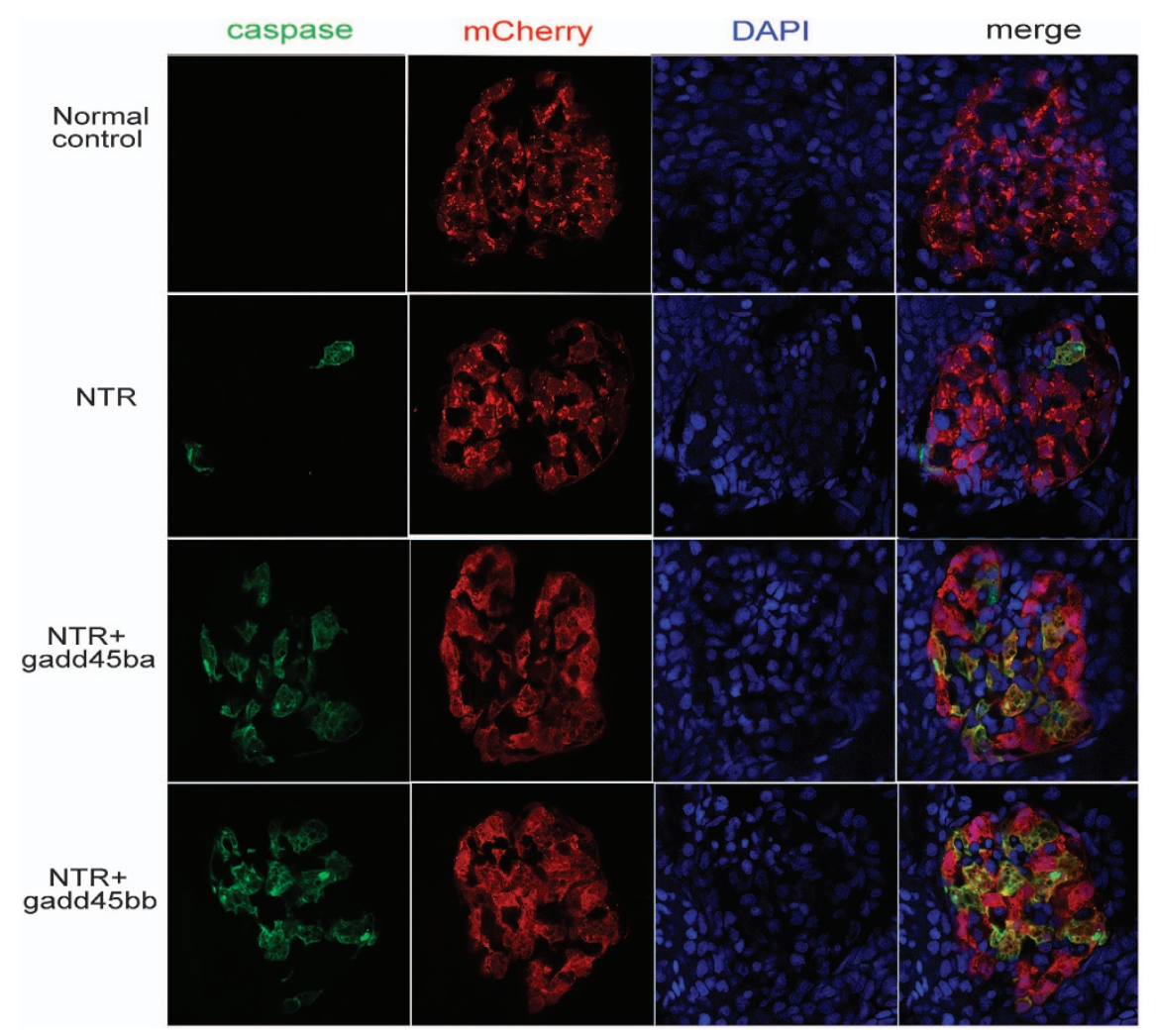

Figure 5 gadd45ba/gadd45bb overexpression aggravated podocyte apoptosis. Confocal images of the pronephric glomeruli in zebrafish embryos were treated with $100 \mu \mathrm{M}$ MTZ from $72 \mathrm{hpf}$ to $96 \mathrm{hpf}$. Green fluorescence represents active caspase-3 staining; podocytes are marked with mCherry red fluorescence and nuclei are labeled with DAPI (original magnification, $\times 630$ ) 
a
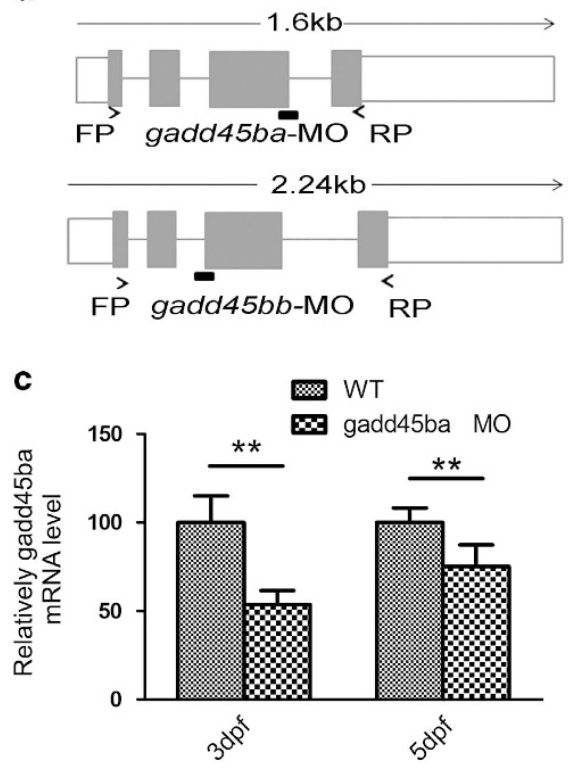

e

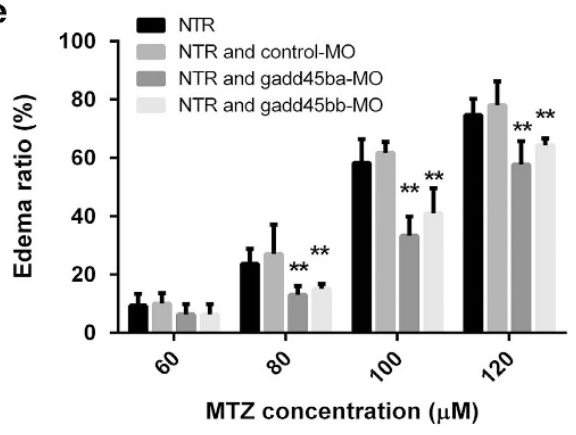

b

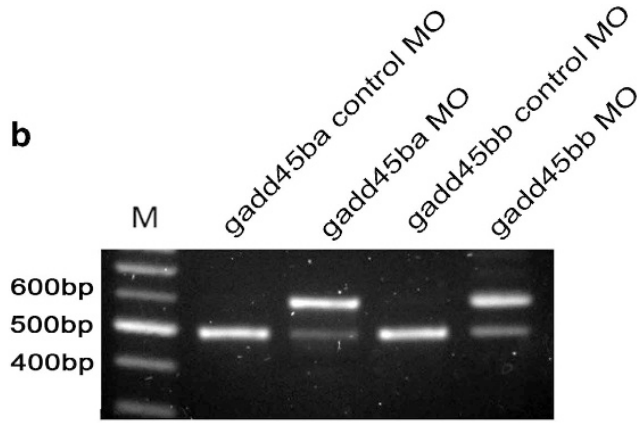

d

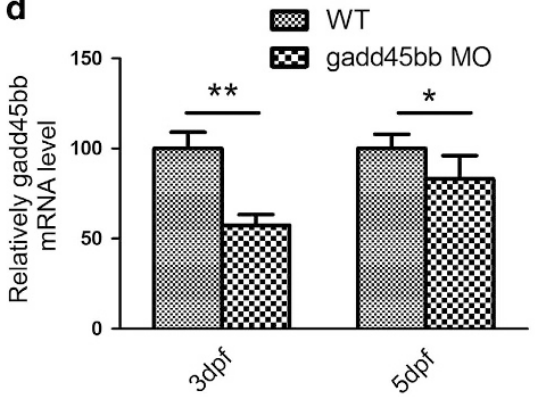

f

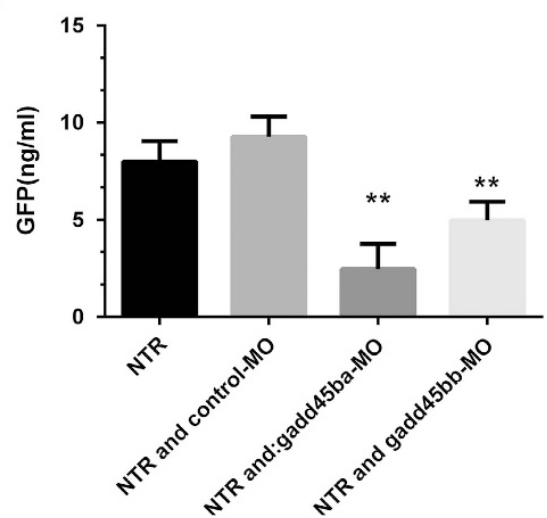

Figure 6 Inhibition of gadd45ba or gadd45bb expression alleviates MTZ-induce edema and proteinuria. (a) Genomic organization of zebrafish gadd45ba and gadd45bb; target sites of the splice morpholinos and positions of primers used to determine the efficacy of MOs. (b) RT-PCR analysis of gadd45ba/bb mRNA in uninjected and gadd45ba/bb-splice-MO-injected embryos at $5 \mathrm{dpf}$. (c, d) qRT-PCR quantify of gadd45ba/bb mRNA level in wild-type fish and gadd45ba/bb-splice-MO-injected embryos at $3 \mathrm{dpf}$ and $5 \mathrm{dpf}$. ${ }^{*} P<0.05,{ }^{*} P<0.01$. (e) Quantitation of the periorbital edema phenotype. (f) Quantitation of proteinuria with ELISA for GFP. ${ }^{\star} P<0.05$, ${ }^{* \star} P<0.01$ versus NTR group

when GADD45B expression was knocked down using the GADD45B shRNA, confirmed by western blot (Figure 7d), TGF- $\beta$ or PAN-induced podocyte apoptosis was significantly decreased compared with shRNA control group or normal control group (Figures $7 f$ and $g$ ).

GADD45B upregulation is associated with ROS generation and P38 MAPK pathway activation in podocytes. Given that GADD45B was upregulated by multiple factors related to oxidative stress in podocytes and that the cytotoxicity of MTZ/NTR system is related to the reduction of the nitro group to nitro radical anion, ${ }^{6}$ we deduced that reactive radicals may be involved in the upregulation of GADD45B in injured podocytes. As overproduction of ROS has been shown to cause podocyte injury induced by PAN toxicity, ${ }^{7-9}$ we tested whether oxidative stress-mediated GADD45B upregulation in PAN-induced podocyte injury. PAN $(50 \mu \mathrm{g} / \mathrm{ml})$ significantly increased ROS generation in podocytes during $30 \mathrm{~min}$ to $12 \mathrm{~h}$ post treatment (Figure 8a), whereas the same PAN treatment induced GADD45B expression by nearly threefold at $12 \mathrm{~h}$ post treatment (Figures $8 \mathrm{~b}$ and $\mathrm{c}$ ). Pretreatment of podocytes with antioxidant $\mathrm{N}$-acetylcysteine (NAC, $10 \mathrm{mmol} / \mathrm{l})$ before PAN exposure led to a significant reduction in GADD45B level (Figures 8b and c), suggesting the production of ROS mediated the upregulation of GADD45B in podocytes.

GADD45B interacts with MAPK upstream kinase MTK1/MEKK4, leading to the activation of p38 or c-jun $\mathrm{N}$-terminal kinase signaling pathways. ${ }^{10,11}$ MAPKs are 
a

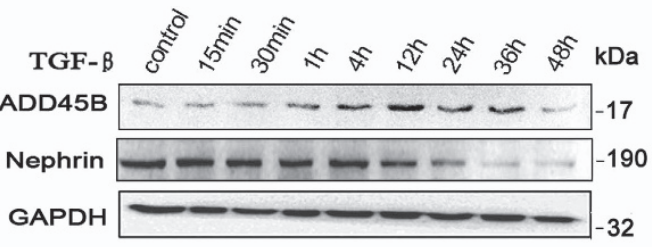

C
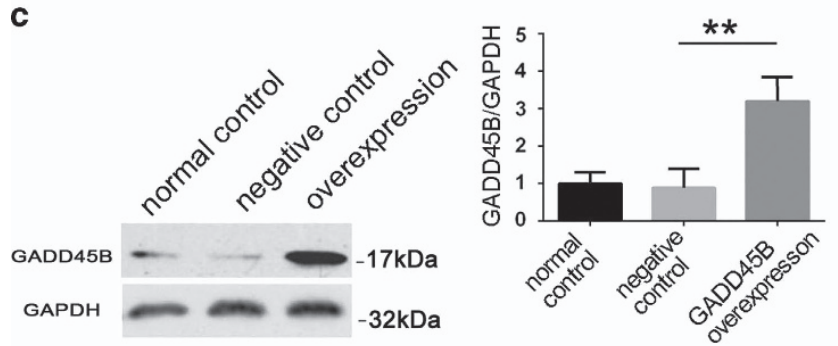

e

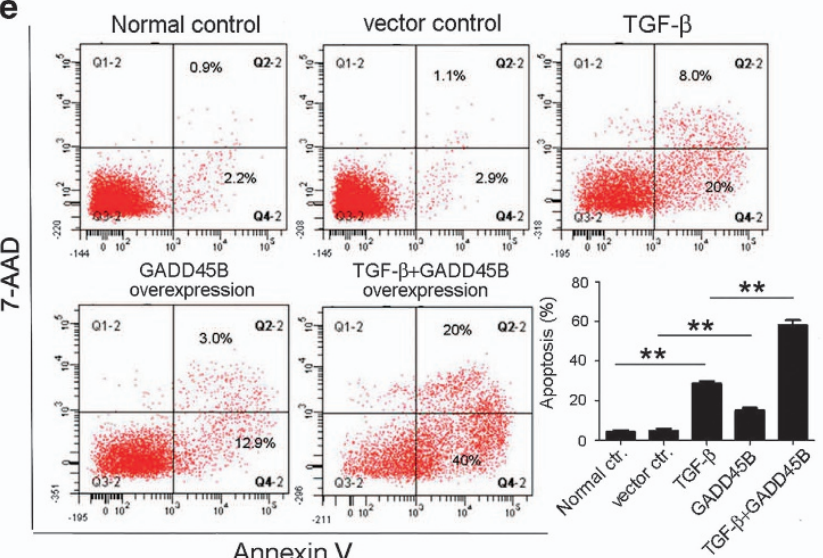

Annexin V

g

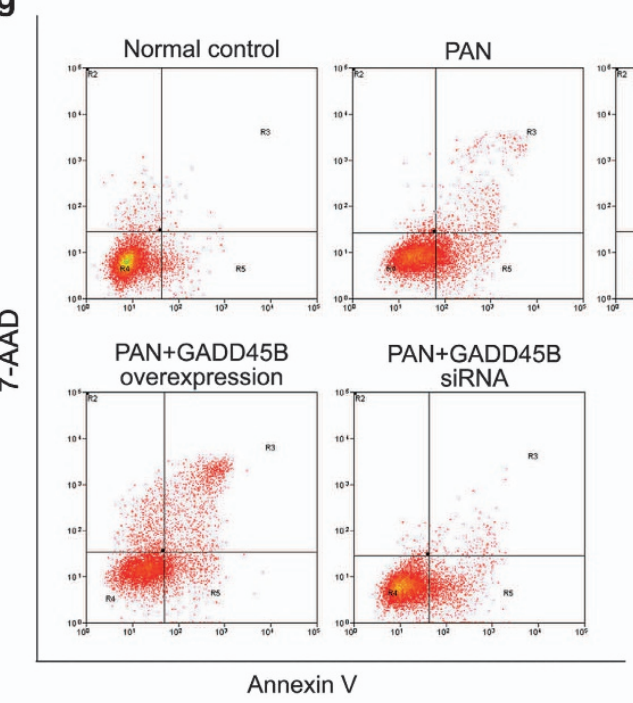

b

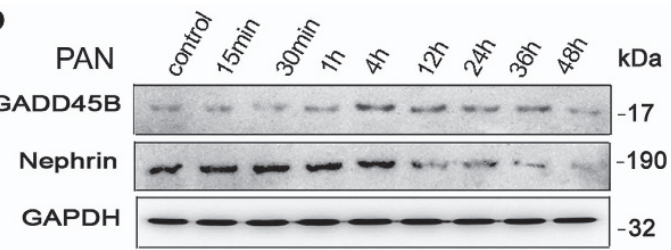

d
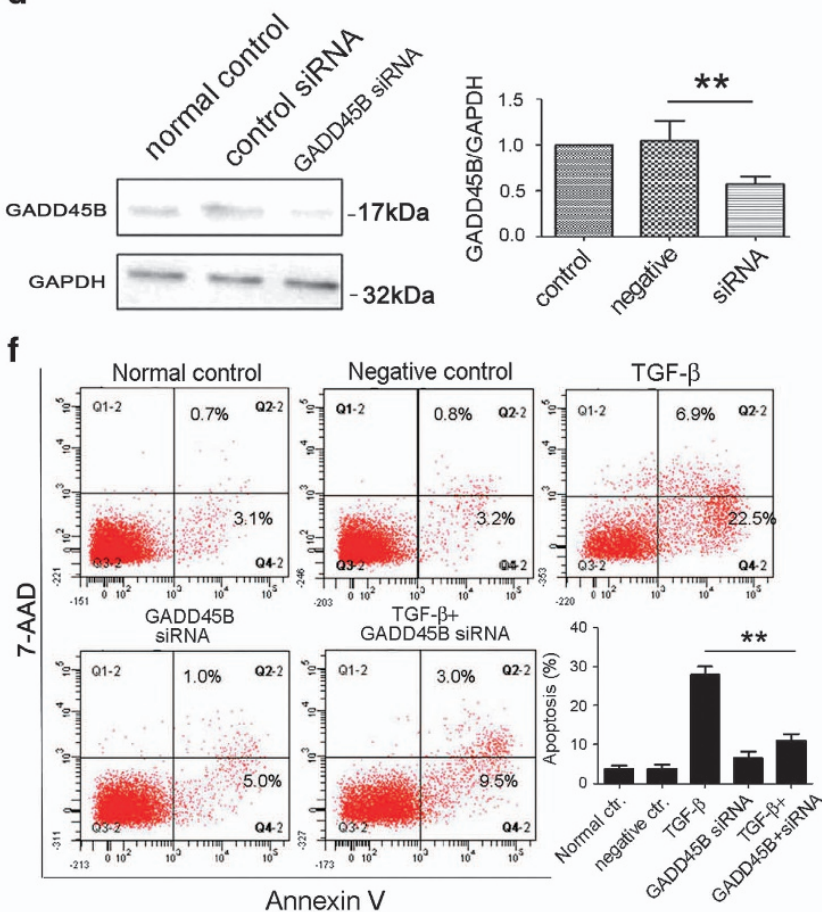

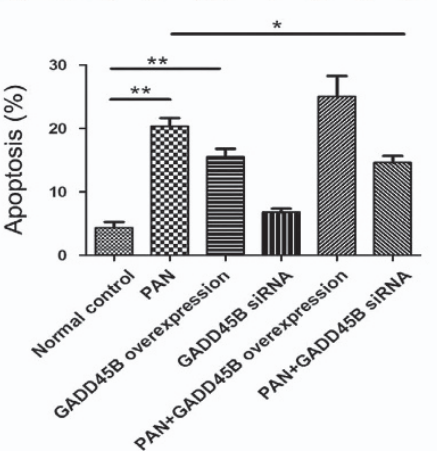

Figure 7 GADD45B expression are upregulated by TGF- $\beta$ and PAN in cultured human podocytes and podocyte apoptosis induced by TGF- $\beta$ is aggravated by GADD45B overexpression and ameliorated by GADD45B knockdown. $(\mathbf{a}, \mathbf{b})$ Western blot analyses of GADD45B and nephrin protein expression upon (a) TGF- $\beta$ (5 ng/ml) and (b) PAN $(50 \mu \mathrm{g} / \mathrm{ml})$ stimulation. Relative protein abundance of each blots was normalized to the gray value of GAPDH. (c, d) Western blot showing GADD45B overexpression (c) or knocked down by shRNA (d) in cultured human podocytes. (e-g) Annexin V staining followed by FCM showing podocyte apoptosis induced by TGF- $\beta$ (e, f) and PAN (g) is aggravate by GADD45B overexpression and ameliorated by GADD45B knockdown. Relative protein abundance of each blot was normalized to the gray value of GAPDH. Results are represented as mean \pm S.D. $(n=3) .{ }^{*} P<0.05,{ }^{* \star} P<0.01$ 
a

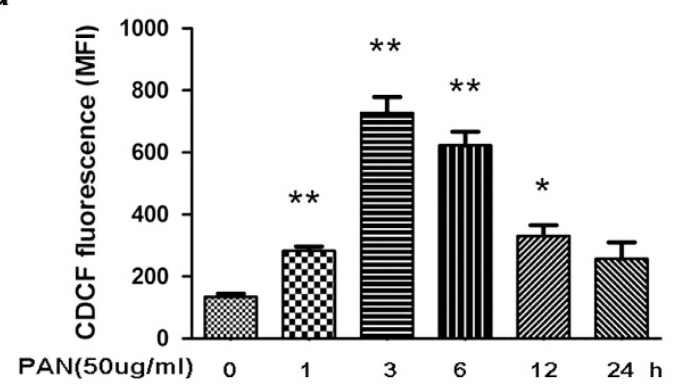

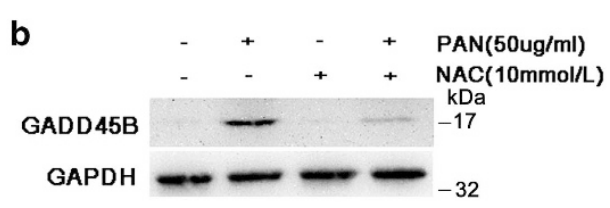

C

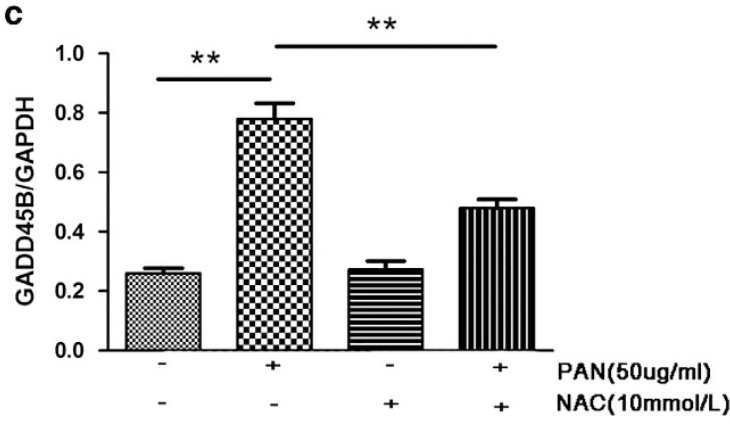

e

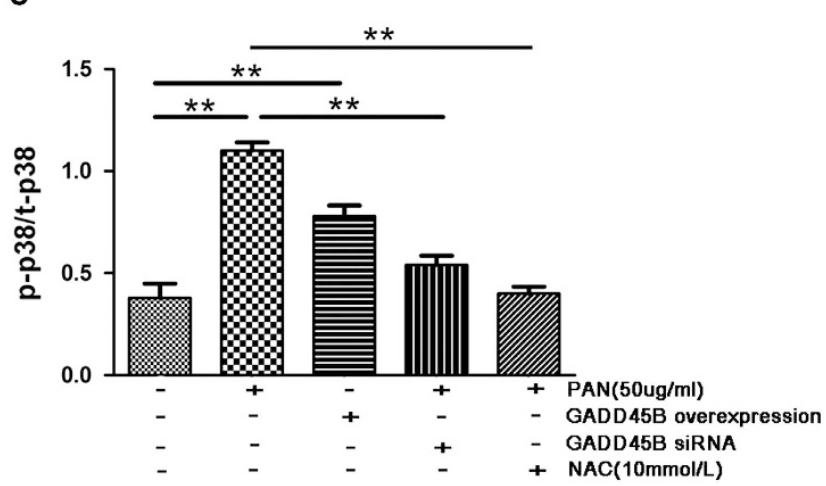

d

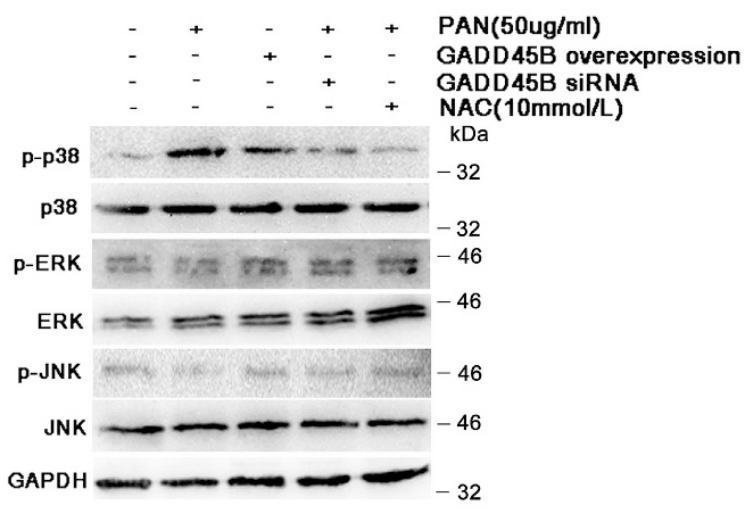

f$$
\text { }
$$

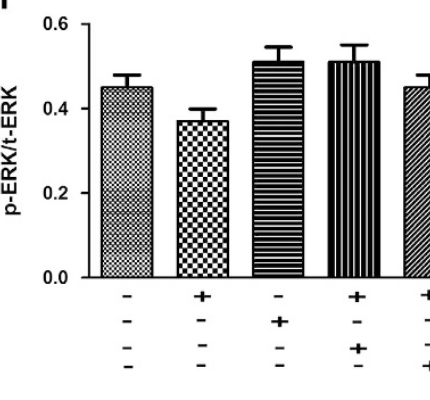

\section{g}

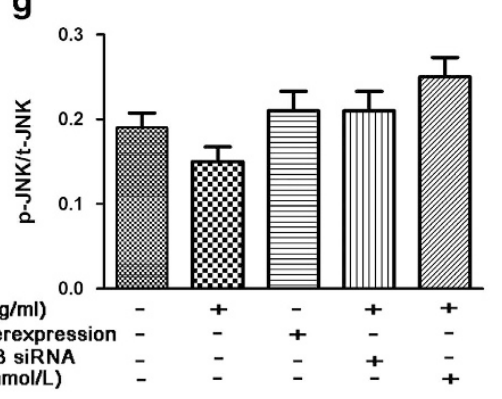

h

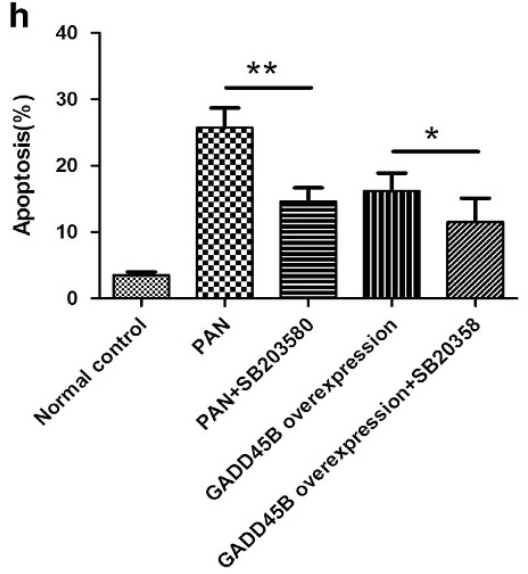

Figure 8 ROS induced by PAN upregulates GADD45B, which subsequently activates p38 MAPK pathway in cultured human podocytes. (a) Time course of PAN-induced cellular ROS generation. (b) Western blot analyses of PAN-induced GADD45B upregulation was diminished by ROS inhibitor NAC. (c) Quantitation of GADD45B protein level in podocytes treated with PAN and NAC. (d) PAN treatment and GADD45B overexpression activates p38 MAPK pathway in cultured podocytes and knockdown of GADD45B inhibits the activation of p38 MAPK pathway by PAN. In contrast, the above treatments have no significant effect on phospho-ERK and phospho-JNK MAPK levels. (e-g) Quantitation of p38(e), ERK(f) and JNK(g) phosphorylation in cultured podocytes (h) p38 inhibitor SB-203580 (25 $\mu$ mol/l) inhibited PAN and GADD45B overexpresson induced podocyte apoptosis. ${ }^{*} P<0.05,{ }^{* \star} P<0.01$. Relative protein abundance of each blot was normalized to the gray value of GAPDH. Results are represented as mean \pm S.D. $(n=3)$. ${ }^{*} P<0.05,{ }^{* *} P<0.01$

known to have crucial roles in the progression of various glomerulopathies, and their inhibition is emerging as a promising therapeutic area for renal diseases. ${ }^{12}$ We found that GADD45B overexpression induced phosphorylation of p38 MAPK in podocytes $(0.75 \pm 0.09$ versus $0.37 \pm 0.10$ normal control, $P=0.008$ ) (Figures $8 d$ and e), but had no effect on activation of JNK and ERK phosphorylation (Figures 8d, $f$ and g). Knockdown of GADD45B expression 
by shRNA diminished PAN-induced p38 MAPK phosphorylation $(0.55 \pm 0.08$ versus $1.13 \pm 0.08$ PAN, $P=0.0009)$. In addition, p38 inhibitor SB-203580 (25 $\mu \mathrm{mol} / \mathrm{l})$ effectively inhibited PAN and GADD45B overexpression induced podocyte apoptosis by $43.1 \pm 9.7 \%$ and $29.1 \pm 5.3 \%$, respectively (Figure 8h). Thus, our data suggest that the ROS-GADD45Bp38 axis mediates the podocyte injury.

\section{Discussion}

GADD45B is among a group of genes upregulated in response to stressful growth arrest or DNA damage. ${ }^{1,13}$ They are involved in many processes during cellular adaptation to a diverse array of cellular stresses, including apoptosis, DNA repair, chromatin regulation and cell cycle delay. ${ }^{1,14-17}$ However, the role of GADD45B in cell stress response is rather complex: it may exert protective or deleterious effects depending on the type of cells and insults. For example, Gupta et $a .^{18}$ found that Gadd45b protected hematopoietic cells from DNA-damaging agents, including ultra violet-induced apoptosis. Larsen et al. ${ }^{19}$ reported that IL-1 $\beta$ stimulated the time-dependent induction of endogenous Gadd45b in rat insulinoma INS-1E cells and rat islets and Gadd45b overexpression can significantly reduce apoptosis induced by IL- $1 \beta$ in INS-1E cells and in mouse beta-TC3 cells. On the other hand, Kim et al. ${ }^{20}$ demonstrated that Gadd45 $\beta$-mediated cardiomyocyte apoptosis under ischemia/anoxia both in cultured H9c2 cells and in the rat heart in vivo. Given the recent studies of a number of animal models of podocyte injury and glomerular diseases implicating GADD45B in podocyte injury, we now have utilized zebrafish models and cultured human podocytes to determine that the induction of GADD45B is an early response to podocyte injury that regulates podocyte apoptosis in response to ROS through the activation of p38 MAPK pathway.

The podocyte function in zebrafish has been proven to be highly conserved in terms of its essential role in maintaining the glomerular filtration barrier. Previous studies of zebrafish orthologs of human nephrosis genes have also demonstrated that these genes have a highly conserved podocyte-specific function across species. ${ }^{21,22}$ Therefore, it is reasonable to speculate the molecular mechanisms underlying podocyte injury responses are also conserved in zebrafish. In this study, we utilized the zebrafish models that we have previously established to facilitate our functional study of GADD45B in podocytes in vivo. ${ }^{5,6}$ In fact, we observed an induction of gadd45ba/b in zebrafish pronephric glomeruli following induced podocyte injury. This is consistent with previously published data from rodent models of podocyte injury ${ }^{3,4}$ and was confirmed in cultured human podocytes injured with various deleterious factors by us. Taking advantage of its convenience and versatility in genetic manipulation, we generated new lines of transgenic zebrafish to demonstrate that overexpression of gadd $45 \mathrm{ba} / \mathrm{b}$ in podocytes exacerbates podocyte apoptosis and proteinuria, whereas inhibition of gadd $45 \mathrm{ba} / \mathrm{b}$ reduces podocyte injury and proteinuria. We designed splice-blocking morpholinos to avoid early embryonic developmental defects due to blocking the function of maternally deposited gadd $45 \mathrm{~b} .^{10}$ This allowed us to test the specific function of injury-induced gadd $45 b$ in podocytes.
Taken together, our zebrafish data not only produce in vivo characterization of gadd45b function in podocytes but also suggest that zebrafish pronephros can be used as an experimental platform for effective functional studies of podocyte-specific genes. Particularly, the toxicity of MTZ as an antibiotic prodrug is mediated by the reduction of the nitro group into protonated one electron nitro radical anion to oxidized DNA, ${ }^{6}$ so our MTZ-induced podocyte injury model is comparable to the conventional podocyte injury models for rodents, such as PAN and adriamycin, which also impose oxidative stress and DNA damage to podocytes. ${ }^{7-9}$ TGF- $\beta$ was also found to affect tubular epithelia cell and kidney fibroblast through oxidative stress. ${ }^{23,24}$

We further explored the mechanism underlying GADD45B induction and subsequent podocyte injury using the in vitro human podocyte culture. Pretreatment of podocytes with antioxidant NAC significantly blocked the induction of GADD45B by PAN, supporting that oxidative stress is correlated with the upregulation of GADD45B in injured podocytes. Moreover, GADD45B overexpression activated p38 MAPK pathway and GADD45B downregulation diminished PAN-induced p38 MAPK activation. P38 inhibitor SB-203580 reduced GADD45B overexpression induced podocyte damage. Among the many kinases activated by ROS, mitogen-activated protein kinases, p38 and c-Jun NH2terminal kinase have been shown to have a key role in oxidative cell injury ${ }^{25,26}$ and p38 activation is crucially involved in podocyte injury, regulating podocyte skeleton disruption, autophagy, apoptosis and so on. ${ }^{27-29}$ Our data established a connection from ROS to p38 activation through GADD45B, elucidating one of the molecular pathways that contribute to podocyte injury.

We identified GADD45B as a new player involved in podocyte stress response in zebrafish as well as in human podocytes. We have also presented successful example of functional analyses of genes involved in podocyte injury using zebrafish models. In particular, with the new genetic tools (CRISPR/Cas9 and TALEN and so on) and transgenic models available for zebrafish podocyte research (inducible podocyte injury and proteinuria assay and so on), the usefulness of this model organism should not be confined to developmental biology of kidney, but rather can be expanded into the study of genetic regulation of podocyte injury and disease.

\section{Materials and Methods}

Fish breeding and maintenance. Zebrafish (Danio rerio) were reared and maintained following standard procedures. ${ }^{30}$ Embryos were collected after natural spawn, kept at $28.5^{\circ} \mathrm{C}$, and staged as hours (hpf) or days (dpf) post fertilization. All procedures were approved by University Committee on Use and Care of Animals at the University of Michigan.

DNA Constructs and transgenic lines. Transgenic fish lines $T g(p o d:$ Gal4), $T g(U A S: g a d d 45 b a, c m / c 2: g f p)$ and $T g(U A S: g a d d 45 b b, c m / c 2: g f p)$ were generated using Tol2 transposon constructs and the Tol2 gateway kit. ${ }^{31,32}$ For pod: Gal4, EGFP was replaced by coding sequence of Gal4 in the pod:EGFP plasmid described previously at the Agel-Clal restriction sites. Danio gadd45ba and gadd45bb cDNA was amplified by RT-PCR from $24 \mathrm{hpf}$ zebrafish embryos. The amplicon were cloned into pME-MCS and sequenced for verification. The primers for gadd45ba are sense: $5^{\prime}$-ttGGTACCatgaccctggaagaagtcgttg-3', antisense: $5^{\prime}$-aGAATTCtcagcgttcttgcagggacag-3'. The primers for gadd $45 \mathrm{bb}$ are sense: 
5'-ttGGTACCatgactctggaggaagttgttg-3', antisense: 5'-aGAATTCtcagcgctcttgcagg-3'. The middle-entry clones, p5E-UAS and p3E-cmlc2:EGFP-pA were assembled using the LR Clonase II plus according to the manufacturer's protocol (Invitrogen, Grand Island, NY). Tg (UAS:NTR-mcherry) was obtained from Zebrafish International Resource Center (ZFIN ID: ZDB-TGCONSTRCT-110215-5). ${ }^{33}$

Microinjection and transgenic fish screening. Expression constructs, together with the Tol2 transposase RNA, were co-injected into one-cell stage AB strain zebrafish embryos as previously described. ${ }^{34}$ The embryos were maintained in egg water in incubator at $28.5^{\circ} \mathrm{C}$. All of the injected embryos were raised to sexual maturity and screened for germline transmission. For detection of $\mathrm{Tg}$ (pod: Gal4) transgenic founder fish, genomic DNA from pools of $F_{1}$ embryos (24 hpf) was isolated accordingly. ${ }^{30}$ PCR was carried out using gene-specific genotyping primers for pod:Gal4: sense:5' - CTGGGAGTGTCGCTACTCTC-3', antisense 5'- CTACATAT CCAGAGCGCCGT-3'; for UAS:NTR-mcherry, sense 5'- ATATACCGGTCAAGCTTA GGCCTCCAAGGC-3', antisense 5'- ATATATCGAT TGGATCCCAAACAGACGCG TC-3'. To identify $T g$ (UAS:gadd45ba:cmlc2:GFP) and $T g$ (UAS:gadd45bb:cmlc2: GFP) transgenic founders, transgenic fish was crossed with wild-type fish $\left(A B^{*}\right)$, $\mathrm{F}_{1}$ embryos (72 hpf) were screened under fluorescence microscopy. Embryos with green fluorescence in heart were raised to adulthood.

$\mathrm{Tg}$ (pod:Gal4) F1 transgenic fish crossed with $\mathrm{Tg}$ (UAS:NTR-mCherry) and raised embryos with mCherry expression in pronephros to adulthood to obtain double transgenic fish Tg(pod:Gal4;UAS:NTR-mcherry).

Podocyte isolation. Kidney from Tg(pod:Gal4;UAS:NTR-mcherry) adult zebrafish were manually dissected and dissociated in $0.5 \%$ trypsin/collagenase. Dissociated cells were then filtered through a $70-\mu \mathrm{m}$ strainer and filtered again through a $30-\mu \mathrm{m}$ strainer. For the purification of podocytes, ${ }^{35}$ kidney single cells were incubated with Anti-mCherry monoclonal antibody (EarthOx, Millbrae, CA, USA) $(1: 200)$ coated Dynabeads pan mouse lgG (Invitrogen dynal, Oslo, Norway) at $4{ }^{\circ} \mathrm{C}$. The positive and negative fractions were collected as mCherry-positive and mCherry-negative fractions, respectively. Isolated cells were placed in RLT Buffer (Qiagen, Hilden, Germany) and RNA was extracted using the RNeasyMicro Kit (Qiagen).

Induction of podocyte injury. Tg(pod:Gal4; UAS:NTR-mcherry) was crossed with $T g$ (UAS:gadd45ba,cmlc2:GFP) or $T g(U A S:$ gadd45bb,cmlc2:GFP). In all, 80hpf embryos were screened with fluorescence microscopy and separated into three groups, (1) embryos with GFP fluorescence (green) in heart and mCherry fluorescence (red) in pronephros. These embryos overexpress both gadd45b and NTR-mCherry in podocytes; (2) embryos with mCherry fluorescence (red) in pronephros only. These embryos expresses NTR-mCherry in podocytes; (3) embryos without fluorescence, this group served as negative control.

Metronidazole (1-[2-hydroxyethyl]-2-methyl-5-nitroimidazole) is reduced by E. coli NTR and converted into a potent DNA interstrand cross-linking molecule, causing cell death. To induce podocyte-specific injury, MTZ was added to E3 medium together with $0.1 \%$ DMSO. Embryos sorted at $80 \mathrm{hpf}$ were treated with MTZ/DMSO at the designated dosages for $48 \mathrm{~h}$. In total, $0.1 \%$ DMSO was used as the vehicle control in all experiments. The experiment design is showed in Figure 7a.

Reverse transcription PCR. Zebrafish Glomeruli were dissected from $\mathrm{Tg}$ (pod:Gal4;UAS-NTR-mCherry) and total RNA was isolated with RNA miniprep (Zymoresearch, Orange County, CA, USA). First-strand cDNAs were synthesized using the Superscript II first-strand synthesis system (Invitrogen), following the manufacturer's protocol. In total, $2 \mu$ of the reserve transcription product was used for PCR with gene-specific primers: for gadd45ba (sense) $5^{\prime}$-ACTGCATCCTCGT CACTAACTC-3' and (antisense) 5'-TTTTGCAACGGCTCTCCTCA-3'; for gadd 45bb (sense) 5'-TGTTACTAACCCCCAAGCCG-3' and (antisense) 5'-GGCAATAGA AGGCACCCAC- $3^{\prime}$. The qRT-PCR analysis to determine the expression levels of gadd45b genes was performed using the Power SYBR Green PCR Master Mix (Applied Biosystems, Rockford, IL, USA). The levels of ef1a were used to normalize the relative mRNA abundance.

Whole-mount in situ hybridization. Whole-mount in situ hybridization using digoxigenin (DIG)-labeled riboprobes was carried out as previously described. ${ }^{36}$ The embryos were fixed with $4 \%$ paraformadehyde (PFA) in phosphate-buffered saline (PBS), hybridized with a DIG-labeled gadd45b RNA probe at $70^{\circ} \mathrm{C}$, followed by incubation with anti-DIG antibody conjugated with alkaline phosphatase and by staining with the substrates, nitro blue tetrazolium and 5-bromo, 4-chloro, 3-indolyl phosphate (NBT/BCIP).
Apoptosis detection by caspase-3 immunostaining. Antibody against cleaved caspase-3 (BD, Franklin Lakes, NJ, USA) was used at 1:500 and secondary antibody was Alexa fluo488 labeled antibody (Invitrogen, Eurgen, OR, USA) at $1: 2000$. The confocal images were captured with a Leica SP5 laser scanning microscope.

Proteinuria assay. Transgenic fish were subjected to the induction of podocyte injury aforementioned at specified developmental stages. To measure GFP leakage with ELISA, 20 embryos per well were placed into 12-well plates at $80 \mathrm{hpf}$ and treated with MTZ in E3 medium for $24 \mathrm{~h}$ and $100 \mathrm{ml}$ of medium was assayed using the GFP ELISA kit (Cell Biolabs, San Diego, CA, USA) following the manufacturer's protocol. Each experiment was repeated for at least three times independently.

Transmission electron microscopy. In all, 96hpf embryos were fixed in cold $3.75 \%$ glutaraldehyde for $4 \mathrm{~h}$ and postfixed in phosphate-buffered $1 \%$ osmium tetroxide for $2 \mathrm{~h}$. Followed by dehydration with graded series of ethanol, the specimens were embedded in Epon 812 Ultrathin sections $(70 \mathrm{~nm})$, were stained with uranyl acetate for $20 \mathrm{~min}$ and subsequently stained with lead citrate for $15 \mathrm{~min}$ at room temperature. The specimens were examined and photographed with Hitachi 7500 transmission electron microscope (Tokyo, Japan).

Morpholino antisense oligonucleotides. Morpholino antisense oligonucleotides (MOs) were designed against splice-blocking sequence of zebrafish gadd $45 \mathrm{ba}$ and gadd45bb. The MOs were obtained from GENE TOOLS, LLC, Philomath, OR, USA. The following sequences of morpholinos were used: gadd45ba (Exon 3 Splice Blocking) 5'-TGTATGTGTCTACTTACAGTGACGA- 3. gadd45bb (Exon 3 Splice Blocking) 5'-ATCCCTGAAGAAGTTGACAAACGCA-3 and Control oligo: 5'CCTCTTACCTCAGTTACAATTTATA-3'. The morpholinos were diluted with the injection solution containing $100 \mathrm{mM} \mathrm{KCl}$ and $10 \mathrm{mM}$ HEPES $(\mathrm{pH} 7.6)$ and were injected at a final concentration of $0.5 \mathrm{mM}$ (gadd45ba-MO) and $0.75 \mathrm{mM}$ (gadd45bb-MO) into one-cell stage embryos. For each morpholino, $>300$ individual embryos were injected. Splice blocking was verified using RT-PCR. The primer sets used were: gadd45ba sense, $5^{\prime}$-ttGGTACCatgacc ctggaagaagtcgttg- $3^{\prime}$, antisense: $5^{\prime}$-aGAATTCtcagcgttcttgcagggacag-3'. gadd45bb sense: $5^{\prime}$ ttGGTACCatgactctggaggaagttgttg- $3^{\prime}$, antisense: $5^{\prime}$ - aGAATTCtcagcgctc ttgcagg-3'. PCR products were gel-purified and sequence verified.

Human podocyte culture, treatment and transfection. Human podocytes were cultured as described. ${ }^{37}$ In brief, cells were grown at the permissive temperature $\left(33^{\circ} \mathrm{C}\right)$ in RPMl 1640 medium supplemented with $10 \%$ fetal bovine serum, insulin-transferrin-selenium, glutamine and penicillin/streptomycin (all from Life Technologies, Carlsbad, CA, USA). To induce differentiation, cells were switched to the non-permissive temperature $\left(37^{\circ} \mathrm{C}\right)$ for 14 days. The podocytes were treated with $5 \mathrm{ng} / \mathrm{ml} \mathrm{TGF}-\beta, 50 \mu \mathrm{g} / \mathrm{ml}$ PAN to induce injury.

GADD 45B stable podocytes transfectants were generated. Full-length GADD45B cDNA was cloned into the pLenti6.3-IRES2-eGFP vector (Invitrogen, Shanghai, China) to generate pLenti6.3-GADD45B-IRES2-eGFP. After confirmed by sequencing, the vector and its helper vectors were mixed and co-transfected into $293 \mathrm{~T}$ cells to obtain recombinant virus containing GADD45B gene, which were used to infect proliferating podocytes. Viruses only carrying eGFP were used as control. Stably transfected podocytes were selected in serial passages using blasticidin $(10 \mu \mathrm{g} / \mathrm{ml})$. Stably transfected podocytes were allowed to differentiate as described above, except in the presence of lower concentrations of blasticidin $(2 \mu \mathrm{g} / \mathrm{ml})$.

Small interfering RNA (siRNA) experiment. Synthetic shRNA targeting human GADD45b and non-targeting control siRNA (Invitrogen) were transfected into differentiated podocytes with Lipofectamine RNAi MAX reagent (Invitrogen, Carlsbad, CA). The target sequences of double-stranded nucleotides used for siRNA knockdown are 5'-ACGAGTCGGCCAAGTTGATGAATGT-3' for GADD45B (1), 5'-CAGTCCTTCTGCTGTGACAACGACA-3' for GADD45B (2), 5'-GAGGTGGC CAGCTACTGCGAAGAAA-3' for GADD45B (3). A nonspecific, red fluorescencelabeled, double-strand RNA (BLOCK-iT Alexa Fluor Red Fluorescent Oligo (Invitrogen), was transfected in parallel as an siRNA-negative control. Synthetic shRNA transfection was performed according to the manufacturer's protocol. At $48 \mathrm{~h}$ after transfection, cells were treated with TGF- $\beta$ and PAN for $24 \mathrm{~h}$. The total protein extracts from the cells were used for western blot analysis. 
Western blot analysis of GADD45B protein expression. Cells were lysed in cold cell lysis buffer ( $50 \mathrm{mM}$ Tris, $150 \mathrm{mM} \mathrm{NaCl}, 10 \mathrm{mM}$ EDTA, $1 \%$ Triton $X-100)$ containing protease and phosphatase inhibitors. The cell lysates $(50 \mu \mathrm{g})$ were heated for $5 \mathrm{~min}$ at $95^{\circ} \mathrm{C}$ in sample buffer, separated on $10 \%$ SDS- polyacrylamide gel and transferred onto nitrocellulose membrane. After blocking with $5 \%$ non-fat dry milk, membranes were probed with rabbit polyclonal antibody to GADD45B and GAPDH (1:1000, Santa Cruz, Waltham, MA, USA). Membranes were incubated with HRP-conjugated anti-rabbit antibody $(1: 1000)$ and visualized by enhanced chemiluninecence detection. Each experiment was repeated for at least three times independently.

Annexin V flow cytometric analysis of apoptosis. After the treatment, podocytes were collected, washed with ice-cold PBS twice, resuspended in $100 \mu \mathrm{l}$ of binding buffer, and then incubated with APC-conjugated Annexin V(1:20) and 7$\operatorname{AAD}(1: 10)$ (Liankebio, Hanzhou, China) at room temperature for $15 \mathrm{~min}$, followed by analysis with FACScan using Cellquest software (Becton Dickinson, Franklin Lakes, NJ, USA). Each experiment was repeated for at least three times independently.

ROS assay. Human podocytes were treated with $50 \mu \mathrm{g} / \mathrm{ml}$ PAN and the intracellular production of ROS was assayed using the fluoroprobe CM-H2DCFDA (chloromethyl-2, -dichlorodihydrofluorescein diacetate as described previously. ${ }^{7}$ Each experiment was repeated for at least three times independently.

Western blot analysis of MAPK activation. MAPK activation was analyzed with western blot as described before. ${ }^{38}$ Primary antibodies were diluted in TTBS and added as follows: anti JNK and anti-phospho-JNK, anti ERK 1/2, anti-phospho-ERK1/2, anti p38, antiphospho-p38 (Santa Cruz Biotechnology, Santa Cruz, CA, USA; mouse monoclonal IgG, dilution 1:200); anti GAPDH (Beyotime Biotechnology; mouse monoclonal, dilution 1: 10 000). Each experiment was repeated for at least three times independently.

Statistical analysis. Statistical analyses were performed with SPSS software (version 11.5). Results were expressed as means \pm S.D. Two groups were compared using the unpaired $t$-test. Comparison of three or more groups was performed by one-way ANOVA. $P<0.05$ was considered statistical significance, and $P<0.01$ was considered highly statistical significance.

\section{Conflict of Interest}

The authors declare no conflict of interest.

Acknowledgements. The project was supported by grants from the National Basic Research Program of China (973 Program, 2012CB517600 and 2012CB517606), the National Nature Science Foundation of China (No. 81470943, No. 81100490), the 'Six Major Talent Peaks' of Jiangsu Province (WSN-079-2013), American Society of Nephrology Carl W Gottschalk Research Scholar Grant and NIH grants (R00DK091405 and P30DK079312) to WZ. The National Nature Science Foundation of China (No. 81570644).

1. Salvador JM, Brown-Clay JD, Fornace Jr AJ . Gadd45 in stress signaling, cell cycle control, and apoptosis. Adv Exp Med Biol 2013; 793: 1-19.

2. Zhang N, Ahsan MH, Zhu L, Sambucetti LC, Purchio AF, West DB. NF-kappaB and not the MAPK signaling pathway regulates GADD45beta expression during acute inflammation. J Biol Chem 2005; 280: 21400-21408.

3. Pippin JW, Durvasula R, Petermann A, Hiromura K, Couser WG, Shankland SJ. DNA damage is a novel response to sublytic complement C5b-9-induced injury in podocytes. J Clin Invest 2003; 111: 877-885.

4. Shi S, Yu L, Chiu C, Sun Y, Chen J, Khitrov G et al. Podocyte-selective deletion of dicer induces proteinuria and glomerulosclerosis. J Am Soc Nephrol 2008; 19: 2159-2169.

5. Zhou W, Hildebrandt F. Inducible podocyte injury and proteinuria in transgenic zebrafish. J Am Soc Nephrol 2012; 23: 1039-1047.

6. Edwards DI. Nitroimidazole drugs-action and resistance mechanisms. I. Mechanisms of action. J Antimicrob Chemother 1993; 31: 9-20.

7. Zheng CX, Chen ZH, Zeng CH, Qin WS, Li LS, Liu ZH. Triptolide protects podocytes from puromycin aminonucleoside induced injury in vivo and in vitro. Kidney Int 2008; 74 : 596-612.
8. Marshall CB, Pippin JW, Krofft RD, Shankland SJ. Puromycin aminonucleoside induces oxidant-dependent DNA damage in podocytes in vitro and in vivo. Kidney Int 2006; 70: 1962-1973.

9. Vega-Warner V, Ransom RF, Vincent AM, Brosius FC, Smoyer WE. Induction of antioxidant enzymes in murine podocytes precedes injury by puromycin aminonucleoside. Kidney Int 2004; 66: 1881-1889.

10. Kawahara A, Che YS, Hanaoka R, Takeda H, Dawid IB. Zebrafish GADD45beta genes are involved in somite segmentation. Proc Natl Acad Sci USA 2005; 102 361-366.

11. Takekawa M, Saito H. A family of stress-inducible GADD45-like proteins mediate activation of the stress-responsive MTK1/MEKK4 MAPKKK. Cell 1998; 95: 521-530.

12. Grande MT, Lopez-Novoa JM. Therapeutical relevance of MAP-kinase inhibitors in renal diseases: current knowledge and future clinical perspectives. Curr Med Chem 2008; 15 2054-2070.

13. Fornace Jr AJ, Alamo Jr I, Hollander MC. DNA damage-inducible transcripts in mammalian cells. Proc Natl Acad Sci USA 1988; 85: 8800-8804.

14. Hoffman B, Liebermann DA. Gadd45 in modulation of solid tumors and leukemia. Adv Exp Med Biol 2013; 793: 21-33.

15. Hoffman B, Liebermann DA. Gadd45 modulation of intrinsic and extrinsic stress responses in myeloid cells. J Cell Physiol 2009; 218: 26-31.

16. Liebermann DA, Hoffman B. Gadd45 in stress signaling. J Mol Signal 2008; 3: 15.

17. Chakravarty D, Cai Q, Ferraris JD, Michea L, Burg MB, Kultz D. Three GADD45 isoforms contribute to hypertonic stress phenotype of murine renal inner medullary cells. Am J Physiol Renal Physiol 2002; 283: F1020-F1029.

18. Gupta M, Gupta SK, Hoffman B, Liebermann DA. Gadd45a and Gadd45b protect hematopoietic cells from UV-induced apoptosis via distinct signaling pathways, including p38 activation and JNK inhibition. J Biol Chem 2006; 281: 17552-17558.

19. Larsen CM, Dossing MG, Papa S, Franzoso G, Billestrup N, Mandrup-Poulsen T. Growth arrest- and DNA-damage-inducible 45beta gene inhibits c-Jun N-terminal kinase and extracellular signal-regulated kinase and decreases IL-1beta-induced apoptosis in insulinproducing INS-1E cells. Diabetologia 2006; 49: 980-989.

20. Kim YA, Kim MY, Yu HY, Mishra SK, Lee JH, Choi KS et al. Gadd45beta is transcriptionally activated by p53 via p38alpha-mediated phosphorylation during myocardial ischemic injury. J Mol Med (Berl) 2013; 91: 1303-1313.

21. Kramer-Zucker AG, Wiessner S, Jensen AM, Drummond IA. Organization of the pronephric filtration apparatus in zebrafish requires Nephrin, Podocin and the FERM domain protein Mosaic eyes. Dev Biol 2005; 285: 316-329.

22. Fukuyo $Y$, Nakamura T, Bubenshchikova E, Powell R, Tsuji T, Janknecht $R$ et al. Nephrin and Podocin functions are highly conserved between the zebrafish pronephros and mammalian metanephros. Mol Med Rep 2014; 9: 457-465.

23. Gentle ME, Shi S, Daehn I, Zhang T, Qi H, Yu L et al. Epithelial cell TGFbeta signaling induces acute tubular injury and interstitial inflammation. J Am Soc Nephrol 2013; 24: 787-799.

24. Bondi $C D$, Manickam N, Lee DY, Block K, Gorin Y, Abboud HE et al. NAD(P)H oxidase mediates TGF-beta1-induced activation of kidney myofibroblasts. J Am Soc Nephrol 2010; 21: 93-102.

25. Forman HJ, Fukuto JM, Torres M. Redox signaling: thiol chemistry defines which reactive oxygen and nitrogen species can act as second messengers. Am J Physiol Cell Physiol 2004; 287: C246-C256

26. Rhyu DY, Yang Y, Ha H, Lee GT, Song JS, Un ST et al. Role of reactive oxygen species in TGF-beta1-induced mitogen-activated protein kinase activation and epithelialmesenchymal transition in renal tubular epithelial cells. J Am Soc Nephrol 2005; 16 : $667-675$.

27. Schiffer M, Bitzer M, Roberts IS, Kopp JB, ten Dijke $P$, Mundel $P$ et al. Apoptosis in podocytes induced by TGF-beta and Smad7. J Clin Invest 2001; 108: 807-816.

28. Jung KY, Chen K, Kretzler M, Wu C. TGF-beta1 regulates the PINCH-1-integrinlinked kinase-alpha-parvin complex in glomerular cells. J Am Soc Nephrol 2007; 18: 66-73.

29. Koshikawa M, Mukoyama M, Mori K, Suganami T, Sawai K, Yoshioka T et al. Role of p38 mitogen-activated protein kinase activation in podocyte injury and proteinuria in experimental nephrotic syndrome. J Am Soc Nephrol 2005; 16: 2690-2701.

30. Westerfield M (ed). The zebrafish book. A guide for the laboratory use of zebrafish (Danio rerio). University of Oregon Press: Eugene, Oregon, 2000.

31. Wan X, Chen Z, Choi WI, Gee HY, Hildebrandt F, Zhou W. Loss of epithelial membrane protein 2 aggravates podocyte injury via upregulation of caveolin-1. J Am Soc Nephrol 2015.

32. Zhou W, Boucher RC, Bollig F, Englert C, Hildebrandt F. Characterization of mesonephric development and regeneration using transgenic zebrafish. Am J Physiol Renal Physiol 2010; 299: F1040-F1047.

33. Pisharath $\mathrm{H}$, Parsons MJ. Nitroreductase-mediated cell ablation in transgenic zebrafish embryos. Methods Mol Biol 2009; 546: 133-143.

34. Kwan KM, Fujimoto E, Grabher C, Mangum BD, Hardy ME, Campbell DS et al. The Tol2kit: a multisite gateway-based construction kit for Tol2 transposon transgenesis constructs. Dev Dyn 2007; 236: 3088-3099. 
35. Anderson BR, Howell DN, Soldano K, Garrett ME, Katsanis N, Telen MJ et al. In vivo modeling implicates APOL1 in nephropathy: evidence for dominant negative effects and epistasis under anemic stress. PLoS Genet 2015; 11: e1005349.

36. Zhou W, Hildebrandt F. Molecular cloning and expression of phospholipase $\mathrm{C}$ epsilon 1 in zebrafish. Gene Expr Patterns 2009; 9: 282-288.

37. Saleem MA, O'Hare MJ, Reiser J, Coward RJ, Inward CD, Farren T et al. A conditionally immortalized human podocyte cell line demonstrating nephrin and podocin expression. J Am Soc Nephrol 2002; 13: 630-638.

38. Chen ZH, Qin WS, Zeng CH, Zheng CX, Hong YM, Lu YZ et al. Triptolide reduces proteinuria in experimental membranous nephropathy and protects against C5b-9-induced podocyte injury in vitro. Kidney Int 2010; 77: 974-988. (c) (i) Cell Death and Disease is an open-access journal published by Nature Publishing Group. This work is licensed under a Creative Commons Attribution 4.0 International License. The images or other third party material in this article are included in the article's Creative Commons license, unless indicated otherwise in the credit line; if the material is not included under the Creative Commons license, users will need to obtain permission from the license holder to reproduce the material. To view a copy of this license, visit http://creativecommons.org/licenses/by/4.0/

Supplementary Information accompanies this paper on Cell Death and Disease website (http://www.nature.com/cddis) 